\title{
Low Voltage Daily Energy Demand Temperature Dependent Representation by Using Circular Statistics
}

\author{
Pucheta Julián'1, Salas Carlos'2, Piumetto Miguel1, \\ Herrera Martín ${ }^{2}$, Rodriguez Rivero Cristian ${ }^{3}$ \\ ${ }^{1}$ Departamento de Electrónica-FCEFyN-Universidad Nacional de Córdoba, Córdoba, Argentina \\ ${ }^{2}$ FTyCA-Universidad Nacional de Catamarca, San Fernando del Valle de Catamarca, Argentina \\ ${ }^{3}$ Center for Neuroscience, University of California at Davis-EEUU, Davis, Argentina \\ Email: jpucheta@unc.edu.ar, calberto.salas@gmail.com, miguel.piumetto@unc.edu.ar, \\ ing_martin_herrera@yahoo.com.ar, crodriguezrivero@ucdavis.edu
}

How to cite this paper: Julián, P., Carlos, S., Miguel, P., Martín, H. and Cristian, R.R. (2019) Low Voltage Daily Energy Demand Temperature Dependent Representation by Using Circular Statistics. Applied Mathematics, 10, 61-74.

https://doi.org/10.4236/am.2019.103006

Received: February 12, 2019

Accepted: March 12, 2019

Published: March 15, 2019

Copyright $\odot 2019$ by author(s) and Scientific Research Publishing Inc. This work is licensed under the Creative Commons Attribution International License (CC BY 4.0).

http://creativecommons.org/licenses/by/4.0/

\begin{abstract}
In this work, a tool that allows visualizing the probability of the power demand according to the temperature and the hours of the day is presented. This aim contributes to the decision making support for the transformer and its service administration. The objective is to represent the demand accurately as a color statistical map based on two variables: the time of day and the ambient temperature. Since the daily energy consumption is periodic regarding the hours of the day in terms of several days, its representation with Gaussian models becomes difficult, but it is simplified when working with circular statistics. The circular statistics used here is the Von Mises distribution, which has the parameters mean address and kappa concentration. Results obtained from measurements made over a year in a medium-voltage transformer with intervals of 60 minutes are shown.
\end{abstract}

\section{Keywords}

Distribution Transformers, Charge Estimation, Time Series, Dynamic Process, Circular Statistics

\section{Introduction}

However, there are factors that affect consumption and therefore infrastructure. As of administration of energy transport ranging from low to medium voltage levels, several studies have been carried out such as prediction of time-series for estimation of the load of the distribution of transformers [1], life power of 
transformers submerged in oil, the correlation of life and temperature [2], the chemical indicators of the degradation of insulating paper in transformers submerged in oil [3], the estimation of lifelong service requested by their insulation [4], and other technologies developed [5] for monitoring of variables in real-time for early-bird warning, alarms and control in the operation of the substation. Mackenzie et al. [6] present some of the recent developments in online-monitoring and diagnostic techniques that help substation's operators know the condition of their transformers and make useful decisions about continuous operation, maintenance or replacement. However, recently in [7] [8], authors propose the use of neural networks for temperature prediction using a lifelong estimation model of the residual life of the transformer.

This proposed system is capable of real time on-line monitoring, early warning, control and alarm for substation operation. Thus, it can be concluded that the problem consisting in making decisions on energy transport networks for home consumption demand is mainly due to inclemency of climate change. Decisions are further intended to improve the reliability of the system, or maintain it within the energy quality standards that regulate the relevant public entity, trying to preserve the installed infrastructure and quality of service. So, there are commitments for an uninterrupted service and a moderate maintenance cost to satisfy the user with an adequate fee.

This problem can be addressed by establishing appropriate models with analysis methodologies that involve real situations through data available to represent the process. In this article, data from measurements obtained from a Control Center (CC) in a Transformer Substation of the EPEC company, distributor of the Cordoba Capital City, Argentina, were used to obtain electrical recording data for a full year from a profile of load. The CC is located in University Campus of the National University of Córdoba. The recordings were taken by using DISCAR S.A company's equipment [9], that consists of a DIMEC concentrator [10] and smart meters DIMET3-P-CT [11].

The meters associated to a DC concentrator perform electrical registers such as, voltage, current, maximum active power, active and reactive energy, frequency and power-factor correction every 15 minutes to the DIMEC concentrator, which in each turn is connected to the control center. In this paper, the problem addressed follows the IEEE Standard C57.91-1995 by which establishes parameters and, the impact of the infrastructure is represented by the transformer's life hours. Therefore, the administrator only requires information adequately processed to make decisions. This work proposes a method based on circular statistics that shows the probable state of charge according to the range of ambient temperature in question, according to the day-time of the day. This work is organized as follows: in Section II the formulation of the problem is made taking into account the analysis of the data that are measured from the transformer by industrial instruments; Section III describes the methodology implemented where circular statistics (Von-Mises distribution) is introduced as an alternative to detect non-repetitive patterns. In Sections IV and V the imple- 
mentation is presented with real data measured for the daily demand and the results obtained. Finally, in Section VI a discussion and conclusions are presented, taking into account after the analysis, the temperature curves for decision making.

\section{Problem Formulation}

The problem consists of making decisions associated with the distribution of power electrical energy in medium and low voltage, maintaining the standards of service quality and simultaneously avoiding the deterioration of the infrastructure, specifically the transformer.

Decisions can be diverse, starting with planning maintenance tasks in the transformer and reaching extreme actions such as the disconnection of loads from it, this at the expense of the service quality and therefore seeks to avoid. In order to make the right decision, it is necessary to have easy to interpret information that represents the working scenario of the transformer, according to the ambient temperature and the time of day. Here a representation of the state of the transformer is proposed by means of a statistical heat map.

The problem is faced by starting with the data analysis that is measured from the transformer by the appropriate instruments [9] [10] [11], which provides load values (volt ampere, VA) and ambient temperature $\left({ }^{\circ} \mathrm{C}\right)$.

The variables are related to each other through the user because when the temperature is located in warm values the user tends to consume more energy. In decision-making according to the power load values and temperature there are recommendations [12] [13] [14] [15] to take into account the deterioration of the transformer as it is subjected to severe working conditions [2] [3] [4]. Therefore, for following such recommendations, the daily demand must be expressed in accordance with the standard [12] [13] [14] [15].

The function that approaches the power load demand called Equivalent load, $E_{L}$, is defined as

$$
E_{L}=\sqrt{\frac{L_{1}^{2} t_{1}+L_{2}^{2} t_{2}+L_{3}^{2} t_{3}+\cdots+L_{N}^{2} t_{N}}{t_{1}+t_{2}+t_{3}+\cdots+t_{N}}},
$$

where $L_{1}, L_{2}, \cdots$ are the load steps in VA, $N$ is the total number of loads considered, $t_{1}, t_{2}, \cdots$ is the duration in hours of each load. For example given a full day, this value is indicated in the legend of Figure 1. However, this number is not used in the standard but parameters that describe the behavior through approximations. The power demand is parameterized by four values as detailed in standard [14] [15], which simplify the power demand function called $K 1, K 2, T 1$ and $T 2$. These parameters are related to the maximum consumption $(K 2)$, the base $(K 1)$, and the duration in hours of each level ( $T 2$ and $T 1)$.

\section{Graph Representation of the Demand Function}

A simple but oversized representation is to take the maximum value of the demand in a cycle of 24 hours, and assume it with a duration of 6 hours for each 
side of the peak, as shown by the red line in Figure 1. To compare with the measured demand, it is used the concept of effective value of the signal that is computed by Equation (1) for the whole day. The approximation suggested by the standards [14] [15] points out the use of four parameters $K 1, K 2, T 1$ and $T 2$ as is shown for a day in Figure 1. These four parameters allow simplifying the description of the consumption function in a piece-wise function containing two steps, as recommended by the standard. Figure 1 shows the measured and approximate demands during a day. Note that an approximation of the daily demand of Equation (1) has an error in the demand effective value by excess and the other approximation has it by default.

Often the decisions are made by analyzing the time $T 2$ [1], but this fact gives an idea of the deterioration that has suffered. For this reason, it is convenient to have more evident information related to the temperature and at the same time to detect possible alarm situations related to the excessive work of the transformer.

Here, a method based on circular statistics that achieves the tuning of the approximate curve by the set $\{K 1, K 2, T 1, T 2\}$ maintaining the same effective value as the measured demand is proposed.

In addition, if the ambient temperature is taken into account, the process must be repeated for each thermal range and build a graphical representation.

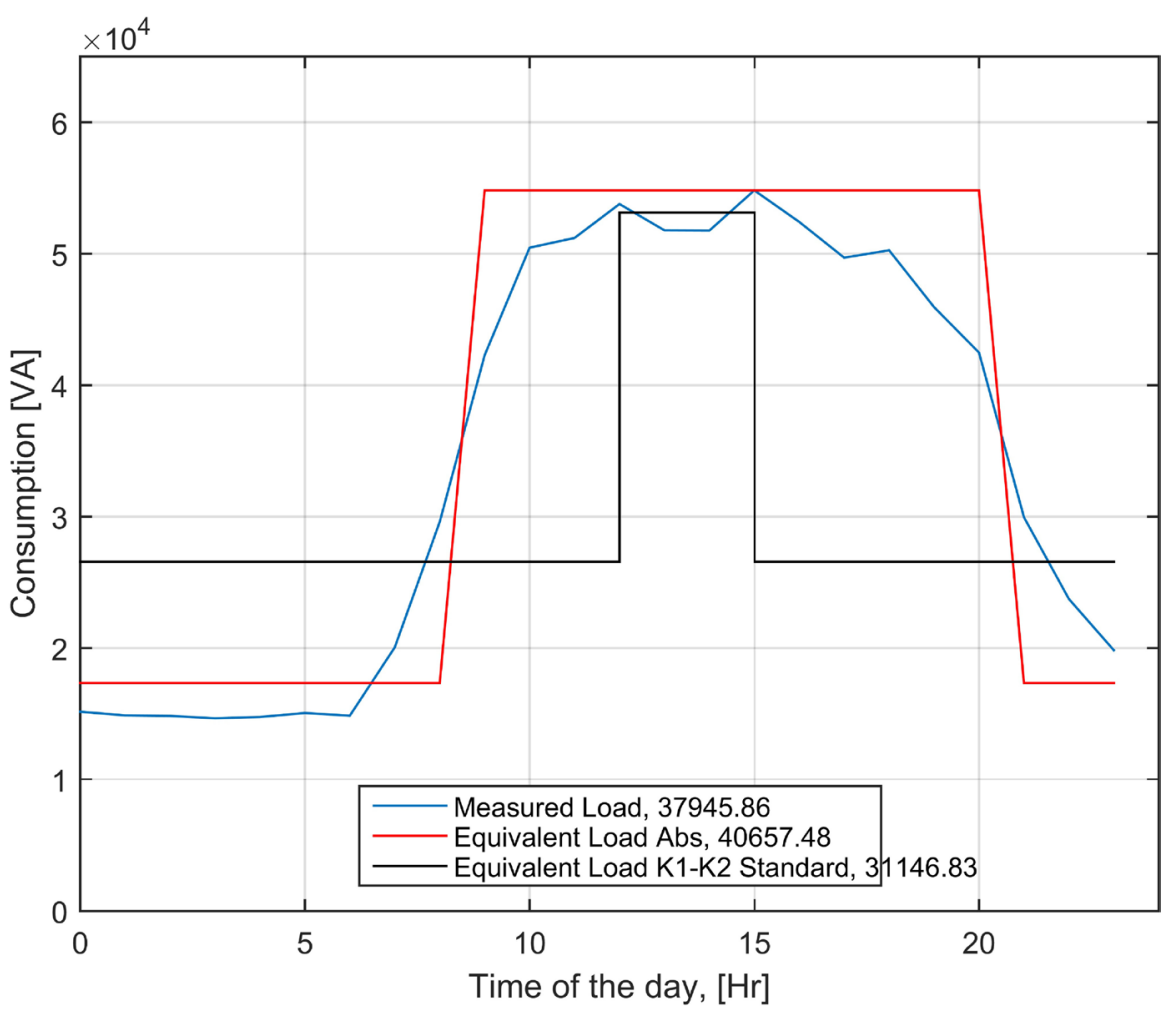

Figure 1. Measured daily power demand 37,945.86 VA computed by Equation (1) and equivalent. For the equivalent demand two calculations are shown: one by taking its maximum value $(40,657.48 \mathrm{VA})$, and another by using the Standard's recommendation $(31,146.83 \mathrm{VA})[14][15]$. 
Note that it is cumbersome if all the ranges overlap, or numerous graphics are generated, which must be mastered simultaneously.

To facilitate the handling of information, this article proposes to generate a methodology for showing the probable charge state as a heat map with two inputs that are the hours of the day and the thermal range.

\section{Purposed Approach}

It is proposed to analyze the power demand according to the time of day, taking the ambient temperature as a parameter and represent a probability distribution function of the power consumption, thus obtaining a three-dimensional graphic or heat map.

Since the daily consumption is a calendar date, and it has a temperature associated with, the interpretation with Gaussian distributions for each hour becomes complex. In this class of problems that are cyclical, their interpretation is simplified when representing temporary variables with circular statistics.

Circular statistics considers the data as points on the unit circle. By this way only the address of the data is of interest. In our case, the address is the time of day, so the unit circle is divided into 24 equal parts and each one is assigned a number of points proportional to the magnitude of the demand.

Once the signal is represented in the unit circle, to use the Von Mises circular distribution, its two parameters must be calculated, which are the average direction and the kappa concentration parameter. With these parameters it is possible to represent the signal according to the distribution of Von Mises, analog to the Gaussian distribution.

\subsection{Circular Statistics Analysis}

In cases such as cyclic calendars processes with repetitions every given time period, problems of Gaussian statistical analysis arise.

The data can be represented by angular measurements giving the orientation or angles in the plane (circular data) or in space (spherical data) [16] [17].

Circular data is the simplest case of this category of data so-called directional data, where the measure is not scalar, but is angular or directional [18].

The basic statistical assumption is that the data is a random sample of a direction population.

To deal with data of this nature it is necessary to use different statistics because the usual statistics used for linear data are inappropriate, given that is difficult to consider the periodic nature of this kind of processes.

The directions in the plane can be observed either as unit vectors in the plane or as points in the unit circle. Therefore, each point $\mathrm{x}$ in the unit circle can be represented by an angle $\theta$ in Cartesian coordinates

$$
x=(\cos (\theta), \operatorname{sen}(\theta)) .
$$

Let $x_{1}, x_{2}, \cdots, x_{n}$ be samples whose corresponding angles are $\theta_{i}, i=1,2, \cdots, n$. 
The average direction $\bar{\theta}$ of $\theta_{1}, \theta_{2}, \cdots, \theta_{n}$ is the direction of $x_{1}+x_{2}+\cdots+x_{n}$ which is the mass center (a.k.a. gravity center) $\bar{x}$ of $x_{1}+x_{2}+\cdots+x_{n}$.

Therefore, if the Cartesian coordinates of $x_{j}$ are $\left(\cos \left(\theta_{j}\right), \operatorname{sen}\left(\theta_{j}\right)\right)$, then $(\bar{C}, \bar{S})$ are the Cartesian coordinates of the mass center computed as

$$
\bar{C}=\frac{1}{n} \sum_{j=1}^{n} \cos \theta_{j}, \quad \bar{S}=\frac{1}{n} \sum_{j=1}^{n} \operatorname{sen} \theta_{j} .
$$

Assuming that $x_{1}, x_{2}, \cdots, x_{n}$ is a nonzero vector, in which case the resulting average length

$$
\bar{R}=\sqrt{\bar{C}^{2}+\bar{S}^{2}}
$$

is greater than zero, then $\bar{\theta}$ is the solution of the following equations

$$
\bar{C}=\bar{R} \cos (\bar{\theta}), \quad \bar{S}=\bar{R} \operatorname{sen}(\bar{\theta})
$$

\subsection{Measures of Concentration and Dispersion}

The resulting average length $\bar{R}$ is the most important measure for dispersion in directional data. Let $x_{1}, x_{2}, \cdots, x_{n}$ be unit vectors, then it is clear that $0 \leq \bar{R} \leq 1$, from which follows that if the directions $\theta_{1}, \theta_{2}, \cdots, \theta_{n}$ are closely grouped, results $\bar{R} \cong 1$; if $\theta_{1}, \theta_{2}, \cdots, \theta_{n}$ are very scattered in the range [0-2 2$]$ then $\bar{R} \cong 0$.

\subsection{Von Mises's Distribution}

The Von Mises's distribution is the most used in the unit circle [16] [18]. Indicated as $v M(\mu, \kappa)$, with parameters $(\mu, \kappa)$ are the distribution center $\mu$ in the interval $[0,2 \pi]$ and the concentration $\kappa$ whose reciprocal is the dispersion, analogous to mean and variance of the Gaussian distribution. The Von Mises's distribution has its density function defined by the expression

$$
g(\theta ; \mu, \kappa)=\frac{1}{2 \pi I_{0}(\kappa)} \exp (\kappa \cos (\theta-\mu))
$$

where $I_{0}$ denotes the modified Bessel function of first type and order 0

$$
I_{0}(\kappa)=\frac{1}{2 \pi} \int_{0}^{2 \pi} \exp (\kappa \cos (\theta)) \mathrm{d} \theta
$$

Note that the Von Mises's distribution is unimodal and is symmetric about $\theta$ $=\mu$. In addition, the mode is for $\theta=\mu$ and the antimode for $\theta=\mu+\pi$. The quotient between the value of density in mode and the value of density in the antimode is given by $\mathrm{e}^{2 \kappa}$, so the higher the value of $\kappa$, the greater the grouping around mode. Observe that the larger the parameter $\kappa$ its density function becomes more concentrated, as indicated in Figure 2.

Note that the distribution deals with periodic data, and places them in the unit circle. The representation of Figure 2 can be done for each working temperature range, and generate a scheme with two variables, one is the temperature and the other the time of day. This makes it an adequate tool to represent the demand for electrical energy and add another dimension to incorporate the temperature 


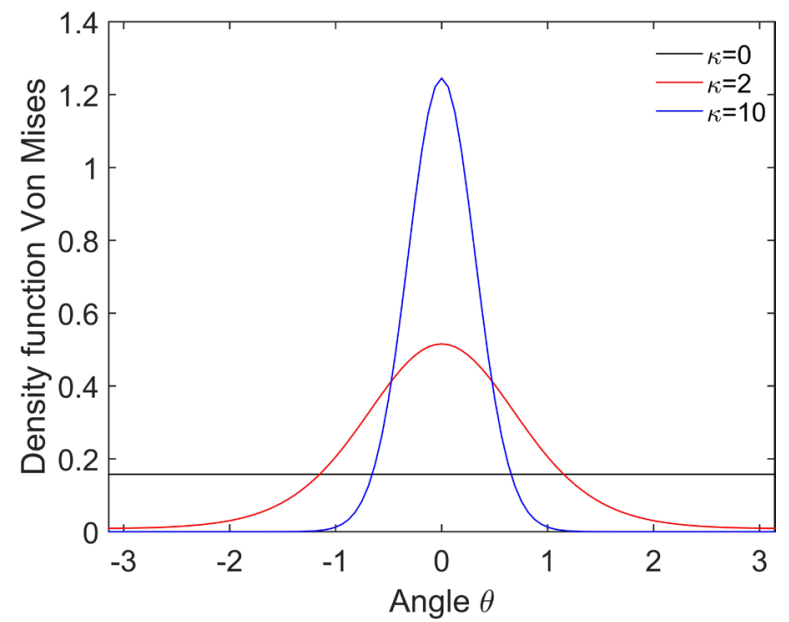

Figure 2. Von Mises distribution for $\mu=0$, as the parameter $\kappa$ increases, the distribution is less flat.

parameter.

\section{Implementation with Measured Data}

Time series of demand values measured during one year are available by acquisitions every 15 minutes obtained through specialized equipment [9] [10] [11].

These measurements are arranged per day and are grouped according to the conditions of maximum temperature of the day, day of the week and season of the year (warm or cold) since it is related to the temperature of the transformer's environment with the measurement.

In accordance to the year's season, ambient temperature ranges are defined for grouping the measurements, given warm season: 15 - 20, 20 - 25, 25 - 30, 30 $35,35-40\left({ }^{\circ} \mathrm{C}\right)$ and cold season: $10-15,15-20,20-25,25-30,30-35\left({ }^{\circ} \mathrm{C}\right)$.

Thus, the data are grouped according to the day of the week and the year's season (Warm or Cold). In this way every Monday, every Tuesday, Wednesday, etc. from a year's season belongs to the same group.

After that each set is divided according to the maximum temperature ranges mentioned. Then each group of day of the week and season of year is divided into five subgroups. From each subgroup a representative day is selected to analyze, in accordance with the highest peak consumption. For the analysis, only representative daily measurements are used.

\subsection{Data Visualization}

Consumption can be represented in different ways with this data, depending on the need to observe the dependence of the demand according to the day of the week, the ambient temperature and the season of year.

For example, real data of the same day with the same temperature range is been processed, and then for a week. Two seasons of temperature, warm and cold, are considered. The power consumption for a Monday whose maximum temperature is in the range $25^{\circ} \mathrm{C}-30^{\circ} \mathrm{C}$ for the two seasons of the year is shown 
in Figure 3.

The analysis of a full week data for the warm season and another of the cold season for the same temperature range is shown in Figure 4, where both weeks are within the range of $30^{\circ}-35^{\circ}$. Note that there is a big change in the consumption between working days and holidays or weekends. So it is inferred that the analysis should be stated when consumption is relevant, as is the case of business days since the rest shows a very low consumption. It should be remembered that these data correspond to a transformer of the National University of Córdoba located in the University City of the City of Córdoba, Argentina.

The consumption peak and the valley for each day of the week are shown in Figure 4. Two data series have been depicted with intention of observe some pattern or signature that serves to characterize it statistically. It can be seen that between business days for the same season a circular distribution like Von Mises's can be proposed [18].

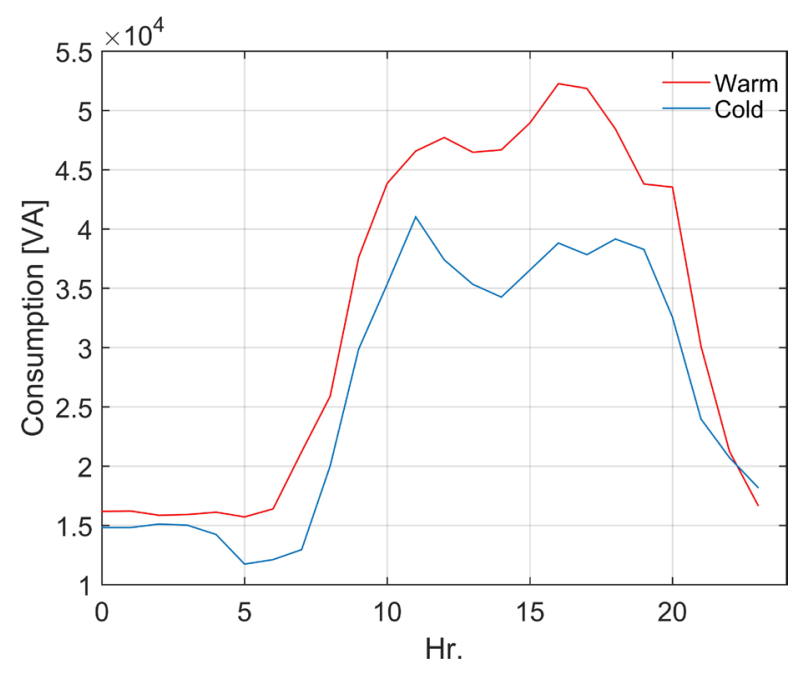

Figure 3. Data of the same day and temperature range $25^{\circ} \mathrm{C}-30^{\circ} \mathrm{C}$ for different seasons.

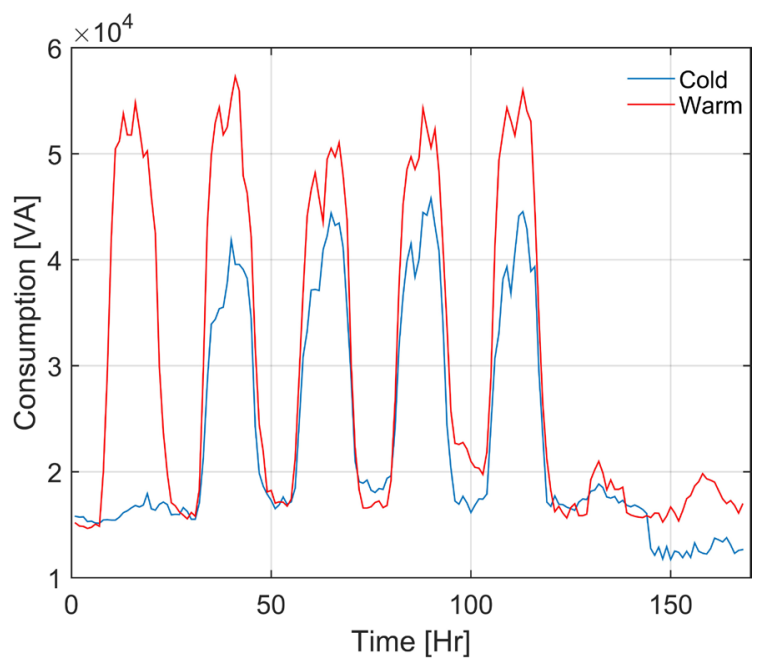

Figure 4. Consumption of two weeks for warm and cold season with a thermal range $30^{\circ}$ $-35^{\circ}$. 


\subsection{Von Mises Probability Density Function for 24 Hours of Demand}

Now, represent the daily consumption using the Von Mises probability density function is proposed, and also show it according to the thermal ranges. To this end, the data must be on the unit circle. Figure 5 shows the process for Von Mises probability density function representation, with a large amount of data that is shown in the unit circle. Note that the data is normalized with its largest value and it must be considered along the analysis.

The result of using the distribution with a single data per hour is also shown, for observing the sensitivity with the amount of data.

The consumption of one day for two seasons is shown in Figure 6. A Wednesdays
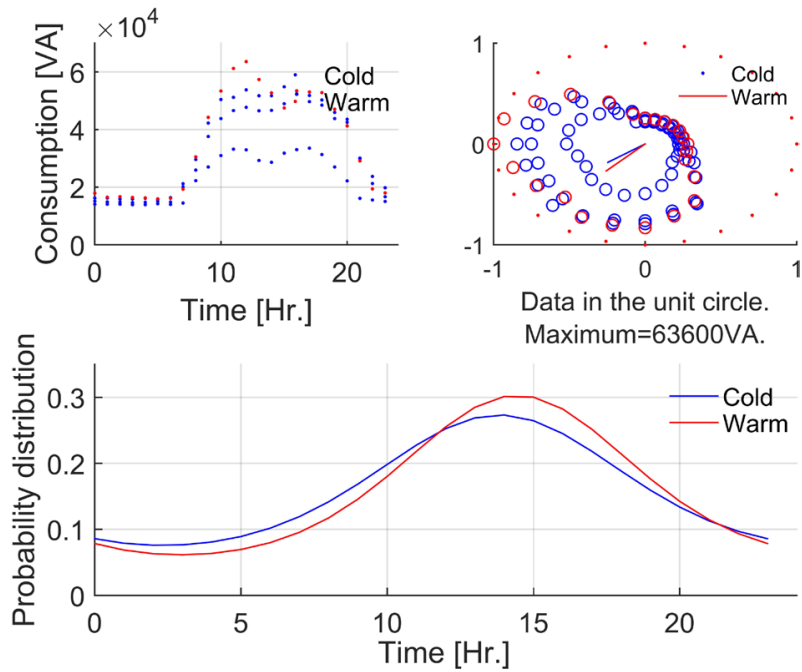

Figure 5. Correspondence of the time data versus its place in the unit circle, and the resulting Von Mises probability density function for two thermal ranges.
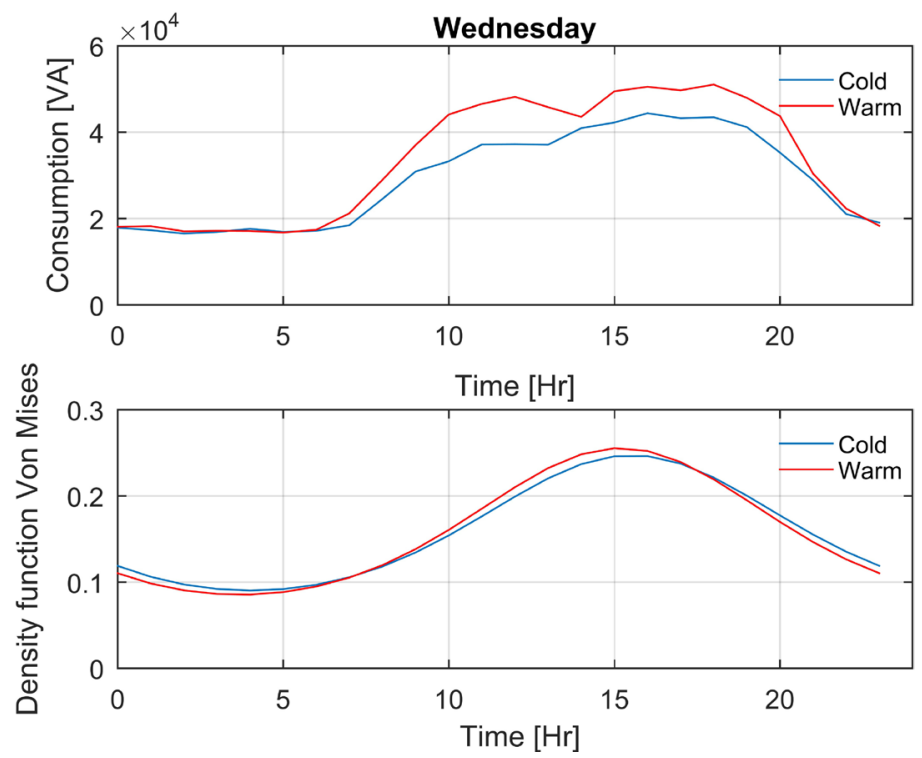

Figure 6. Comparison by Von Mises's distribution of two days Wednesday of the same thermal range and different seasons. 
of warm and cold season, together with their Von Mises's distributions. Note that as the peak consumption increases, the probability density increases at the time the peak occurs.

The time at which the peak of probability density appears is related to the moments of greatest consumption. Thus, the same graph can be compared in Figure 6 on two different days to see the change in the Von Mises's distribution as a function of consumption, which should be read as the distribution of probabilities of maximum consumption.

In Figure 6 the red curve represents the consumption during a day Wednesday of the warm season in the range temperatures $30^{\circ} \mathrm{C}-35^{\circ} \mathrm{C}$, and the blue one on Wednesday in the same range but cold season.

Observe that the demand for the highest temperature has a higher consumption peak, an effect that is also noted in the higher peak of its probability density function.

The distribution of a non-working day, such as Sunday, is shown in Figure 7, where it can be seen that the distribution has a significant quantitative change, since the consumption peak is at 3:00 pm for the warm season in the Wednesday gives 0.25 and on Sunday gives 0.165 at the same time on both days. Note that the trajectories describing the consumptions are very different but not those that describe the Von Mises distributions. The form is maintained with a mode and a dispersion, with its respective scale which facilitates the behavior interpretation of the demand.

\subsection{Using Von Mises for Daily Demand}

The average square value of the demand is explicit by Equation (1) for a single
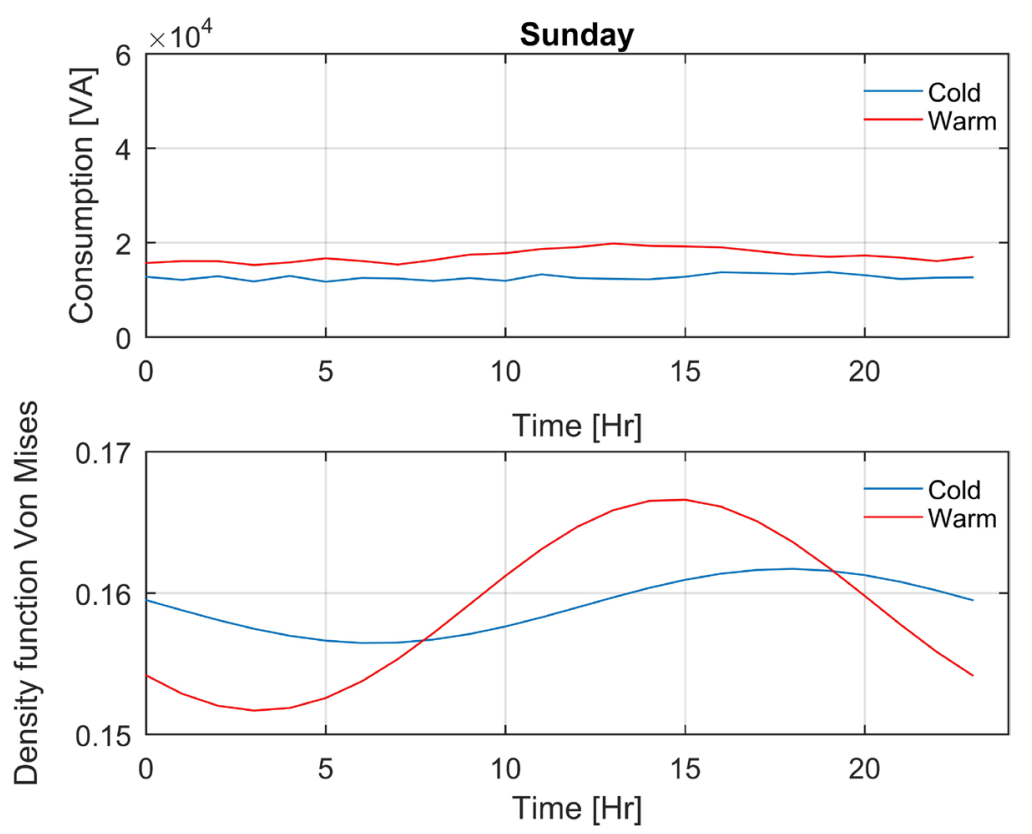

Figure 7. Comparison by Von Mises's distribution of two days Sunday of the same thermal range and different seasons. 
day. This value is final and, of course, loses the information of the maximum value and the valley value that the demand has in the 24 hours.

A representation recommended by the standard shows by Figure 1 in black and another used in practice in red. In this section, a representation of the demand with the same tuple $\left\{K_{1}, K_{2}, T_{1}, T_{2}\right\}$ based on the distribution of Von Mises is generated.

To compute the parameters $\left\{K_{1}, K_{2}, T_{1}, T_{2}\right\}$, the peak of the Von Mises likelihood function detailed in the previous section is determined. The user assigns a percentage between $50 \%$ and $90 \%$ to this value that covers all available measurements.

Then, this value is been compared with the likelihood function hour to hour and the hours in which the likelihood function is greater than the percentage of the maximum are the hours that define $T_{2}$. Once the hours that make up $T_{2}$ are defined, $K_{2}$ is calculated according to [15] by

$$
K_{2}=\frac{1}{T_{2}} \sqrt{\sum_{t=i}^{++T_{2}} W_{t}^{2}} .
$$

The consumption squares are added for the hours specified by $T_{2}$ ( $i$ is the first hour whose likelihood function exceeds the percentage of the maximum previously determined), the square root of that sum is calculated and divided by $T_{2}$. $T_{1}$ is composed of all hours that do not form $T_{2}$, and $K_{1}$ is calculated equal that $K_{2}$ but considering the demand only in the hours that make up $T_{1}$. In Figure 8 the approximation is shown using a percentage of $50 \%$ in green.

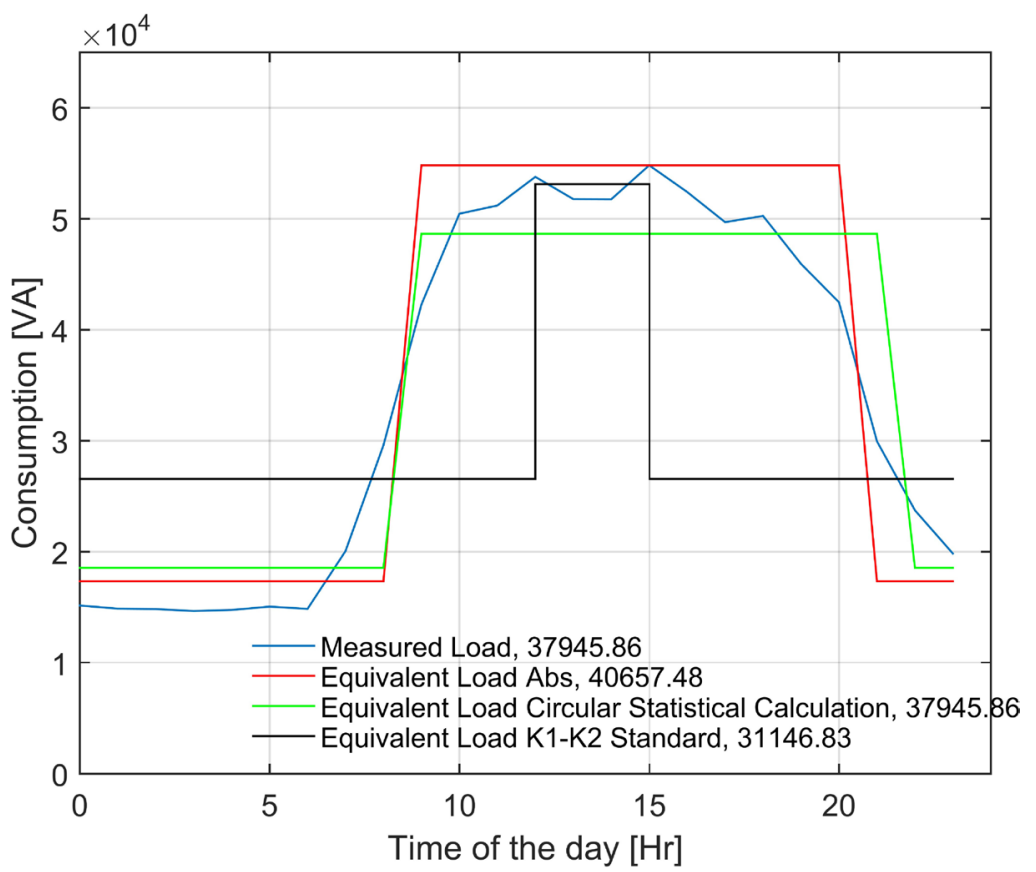

Figure 8. Measured daily demand (37,945.86 VA) and equivalent. For the equivalent demand three calculations were added, which are by taking maximum value (40,657.48 VA), by using the statistical computation and by using the Standard recommendation [14] [15] $(31,146.83 \mathrm{VA})$. 
The daily demand measured by the equipment is shown in blue. Thus, for the equivalent demand, three more calculations are shown, which result from taking the maximum value (red), by using the circular statistical computation (green), and by using the mechanism of the Standard (black) IEEE [15].

Note that there are different criteria to represent the daily demand of a transformer in low voltage and intends to represent the same feature.

Decisions on the transformer are taken based on the value that the set $\left\{K_{1}, K_{2}\right.$, $\left.T_{1}, T_{2}\right\}$ takes to determine its remaining life and to establish maintenance or testing programs.

Note that depending on how the set $\left\{K_{1}, K_{2}, T_{1}, T_{2}\right\}$ is taken, different values for daily power consumption are obtained as show Figure 8.

The set obtained by the circular statistical method is the closest to the mean square value effectively measured, as indicated by the calculation of the daily consumption indicated in the legend.

However, to facilitate the observation and detection of possible transformer overloads, it is more intuitive to observe the load as a heat map with hourly time and temperature inputs.

\section{Obtained Results}

The three-dimensional representation of consumption to facilitate the detection of critical points and valleys in consumption for real values is proposed to perform. To achieve a direct representation of the dependence of consumption with temperature, the Von Mises curves are plotted corresponding to the temperature ranges of a given season (warm or cold).

The results are shown in Figure 9, where by a heat map is represented the

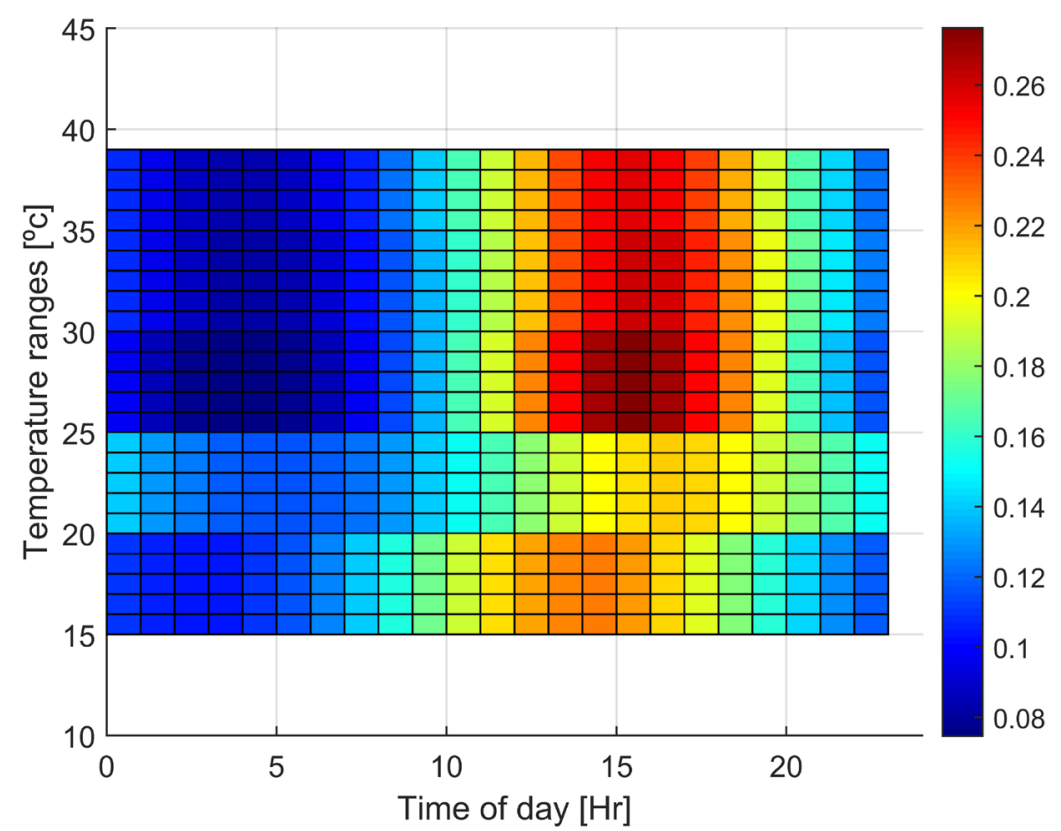

Figure 9. Heat map generated by the purposed method by Von Mises statistics corresponding to the temperature ranges of the selected season. 
correspondence between the maximum consumption likelihood and the ambient temperature range of the transformer under study, in the time band from 0 to 24 hours, obtained using the proposed monitoring tool.

By observing the monitored temperature curves, two areas of importance in the generation of heat determined by color are shown.

An increase in the heat temperature of the due to the season and the time of the day can be observed.

Although the ambient temperature is high within the hourly range, the presence of higher consumption is reflected differently for the selected season.

The mechanism to read this result by the user is as follows. A specific hour and temperature of operation must be established and so to observe what is the likelihood that the consumption is maximum.

\section{Conclusions}

In this paper, a methodology based on real data to model the demand in medium and low voltage transformers by circular statistic of Von Mises probability density was detailed.

A statistical heat map showing the occurrence likelihood of the maximum power in a plane of day time and temperature range is achieved.

In the obtained results, the value of the maximum power consumption is parameterized and must be taken into account together with the probability density.

The results presented were obtained from a transformer in service of the University City of Córdoba (Argentina). The results are a heat map real time updatable that facilitates decision making and simplifies the representation of long time series of two variables in a single graph easy to interpret.

\section{Conflicts of Interest}

The authors declare no conflicts of interest regarding the publication of this paper.

\section{References}

[1] Piumetto, M., Pucheta, J., Vaschetti, J., Gómez Targarona, J.C., Coyos, C. and Arcurio, J. (2017) Predicción por series temporales aplicada a la estimación de cargabilidad de transformadores de distribución para la toma de decisiones. XII Latin American Congress on Electricity Generation and Transmission CLAGTEE 2017. 12 al 15 de noviembre de, Mar del Plata, Argentina.

[2] Flores, W., Mombello, E., Rattá, G. and Jardini, J.A. (2007) Vida de transformadores de potencia sumergidos en aceite: Situación actual. Parte I. Correlación la vida y la temperatura. IEEE Latin America Transactions, 5, 50-54. https://doi.org/10.1109/T-LA.2007.4444533

[3] Emsley, A.M. and Stevens, G.C. (1994) Review of Chemical Indicators of Degradation of Cellulosic Electrical Paper Insulation in Oil-Filled Transformers. IEE Proceedings-Science, Measurement and Technology, 141, 324-334.

[4] Pettersson, L. (1990) Estimation of the Remaining Service Life of Power Transfor- 
mers and Their Insulation. Electra, 133, 65-71.

[5] Pérez, R., Torres, H., Fernández, E. and Fernández, S. (2012) Sistema de Monitoreo en Tiempo Real para el Diagnóstico de Transformadores de Potencia en una Empresa de Energía Eléctrica. 10th Latin Amercican and Caribbean Conference Form Engineering and Techonology, Panamá City, July 23-27 2012, Paper 69.

[6] Mackenzie, E.A., Crossey, J., dePablo A. and Ferguson, W. (2010) On-Line Monitoring and Diagnostics for Power Transformers. 2010 IEEE International Symposium on Electrical Insulation, San Diego, CA, 6-9 June 2010, 1-5.

[7] Wang, T., Wang, Q.G., Wang, P. and Zhang, Y.X. (2017) Thermal Monitoring and Reliability Analysis System for Underground Substation. 2017 IEEE Power \& Energy Society General Meeting, Chicago, IL, 16-20 July 2017, 1-6. https://doi.org/10.1109/PESGM.2017.8273884

[8] Pérez, R., Torres, H., Fernández, E. and Fernández, S. (2012) Sistema de Monitoreo en Tiempo Real para el Diagnóstico de Transformadores de Potencia en una Empresa de Energía Eléctrica. 10 th Latin American and Caribbean Conference for Engineering and Technology, Panamá City, 24-27 July 2012, Paper 6.

[9] http://www.discar.com/

[10] http://www.discar.com/?page_id=135

[11] http://www.discar.com/?page_id=292

[12] AENOR, UNE-IEC 60076-7 (2010) Parte 7: Guía de carga para transformadores potencia sumergidos en aceite.

[13] AENOR, UNE-IEC 60076-2 (2013) Parte 2: Calentamiento de transformadores sumergidos en aceite.

[14] International Standar, IEC 60354 (1991) Loading Guide for Oil-Immerse for Power Transformer. Second Edition.

[15] IEEE Standards, IEEE Std C57.91-1995 (1995) Guide for Loading Mineral Oil Immersed Transformers.

[16] Evans, Hastings, and Peacock (1993) Statistical Distributions. 2nd. Edition, John Wiley and Sons, Hoboken, Chapter 39.

[17] Borradaile, G. (2003) Statistics of Earth Science Data. Springer, Berlin. https://doi.org/10.1007/978-3-662-05223-5

[18] Fisher, N.I. (1993) Statistical Analysis of Circular Data. Cambridge University Press, Cambridge. 